NOUVELLE

\section{Le recyclage de STING par COPA freine I'inflammation}

Alice Lepelley ${ }^{1}$, Marie-Louise Frémond ${ }^{1,2}$
> La première étape de la réponse antivirale passant par les interférons de type I (IFN) est la reconnaissance des acides nucléiques étrangers par les récepteurs de l'immunité innée. Lorsque le récepteur cytosolique d'ADN double brin, cGAS (cyclic AMP-GMP synthase), est activé par son ligand, il synthétise le dinucléotide GMP-AMP cyclique (cGAMP), un second messager qui stimule la protéine adaptatrice STING (stimulator of interferon gene), permettant la phosphorylation de TBKI (TANK-binding kinase 1 ) et IRF3 (interferon regulatory factor 3 ), puis la production d'IFN [1]. Si les IFN ont un rôle protecteur contre les infections virales, il est désormais établi qu'une sécrétion continue et inappropriée d'IFN est toxique pour les tissus. Le concept d'« interféronopathie», formulé en 2011 par Yanick Crow, regroupe les maladies génétiques dues à une activation constitutive des voies des IFN [2].

\section{Le syndrome COPA : des similitudes avec le SAVI}

L'une de ces interféronopathies est une maladie auto-inflammatoire connue sous le nom de STING-associated vasculopathy with onset in infancy (SAVI). Elle est due à des mutations activatrices du gène STINGI (stimulator of interferon gene 1) codant la protéine STING, et comprend une vascularite cutanée, une inflammation systémique et une atteinte interstitielle pulmonaire sévère $[3,4]$. Une autre maladie auto-inflammatoire rare, comportant également une atteinte pulmonaire et ayant des similitudes avec le SAVI, est causée par des mutations, à l'état hétérozygote, du gène COPA, qui code la sous-unité $\alpha$ du coatomère ${ }^{1}$ (COPA) [8] (Figure IA). Cette maladie se caractérise par une pénétrance incomplète ${ }^{2}$. L'expression accrue de gènes stimulés par les IFN, souvent désignée par le terme «signature IFN », est caractéristique des patients ayant une interféronopathie telle que le SAVI [3, 4], et une signature du même type a été identifiée dans le sang de cinq patients COPA [5]. Nous avons alors fait I'hypothèse que le syndrome COPA était une nouvelle interféronopathie, et que STING était impliqué dans sa pathogenèse. Nous avons étudié un groupe de neuf patients atteints du syndrome COPA et confirmé la similitude clinique de ce syndrome avec le SAVI. Nous avons ensuite comparé la biopsie pulmonaire d'une patiente COPA et celle d'une patiente SAVI précédemment décrite [4], et avons observé des lésions histologiques similaires, renforçant l'hypothèse d'un mécanisme pathologique commun. Enfin, nous avons montré la persistance d'une signature IFN et des taux sériques élevés d'interféron $\alpha$ par la technique ultrasensible de dosage Simoa (single molecule array) chez les patients COPA symptomatiques, témoignant d'une activation constitutive de la voie IFN. Les porteurs asymptomatiques de mutations de COPA ont une activation moindre ou absente de cette voie, ce qui suggère qu'une contribution de facteurs (épi)génétiques ou environnementaux supplémentaires est nécessaire à son activation constitutive.

\footnotetext{
${ }^{1}$ Complexe protéique recouvrant les vésicules et permettant leur transport dans la cellule.

${ }^{2}$ Définie pour les maladies monogéniques dominantes, la pénétrance d'une mutation désigne la probabilité, pour une personne porteuse de la mutation, d'être atteinte par la maladie. La pénétrance est dite incomplète lorsque cette probabilité est inférieure à 1 .
}

${ }^{1}$ Université de Paris, Institut Imagine, Laboratoire de neurogénétique et neuroinflammation,

24 boulevard du Montparnasse, 75015 Paris, France.

'Unité d'immuno-hématologie et rhumatologie pédiatriques, Centre de référence maladie rare RAISE, Hôpital Necker-Enfants malades, AP-HP. Centre - Université de Paris, 75015 Paris, France.

alice.lepelley@institutimagine.org marie-louise.fremond@aphp.fr

Les mutations de COPA induisent la signalisation de l'interféron via STING par un effet dominant négatif Pour comprendre le mécanisme d'activation des IFN dans le syndrome COPA, nous avons utilisé un modèle de cellules exprimant COPA de manière endogène mais non STING, que nous avons co-transfectées pour qu'elles produisent COPA, avec ou sans mutation, et STING. Les formes mutées de la protéine COPA induisent une activation de la voie IFN, dont témoigne l'expression du gène codant I'IFN- $\beta$ et des gènes stimulés par les IFN, uniquement en cas de co-transfection avec STING (Figure 1B). Cette activation est donc dépendante de la présence de STING. Elle est par ailleurs inexistante pour la forme de COPA dépourvue de mutation, y compris en présence de STING. Toutes les mutations de COPA décrites chez les patients sont présentes à l'état hétérozygote, et l'expression de ces formes mutées de COPA par transfection dans des cellules produisant déjà la protéine de manière endogène modifie le fonctionnement de ces cellules, ce qui exclut la possibilité qu'une simple haploinsuffisance ${ }^{3}$ rende compte de l'effet de ces mutations chez les patients. Nous avons voulu déterminer si elles agissaient par gain de fonction de l'allèle muté ou par un effet dominant négatif sur l'allèle normal. Dans la lignée de cellules monocytaires THP-1, possédant une voie cGAS-STING fonctionnelle,

\footnotetext{
3 Situation dans laquelle, en présence d'une mutation qui neutralise l'un des deux allèles d'un gène, le produit de l'autre allèle, bien qu'actif, est synthétisé en quantité insuffisante pour permettre le fonctionnement normal des cellules qui l'expriment.
} 
A

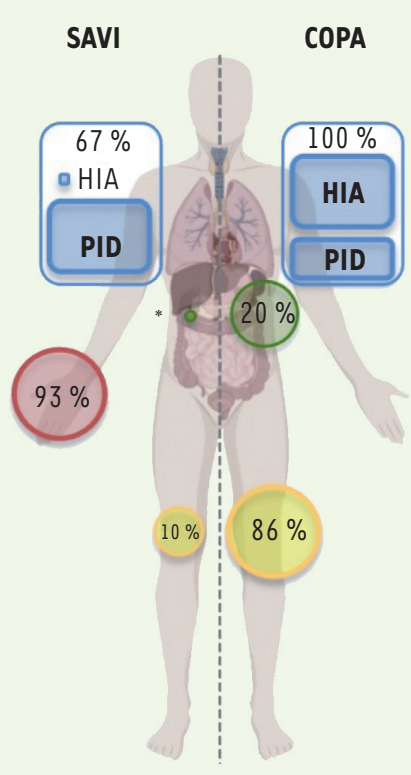

C

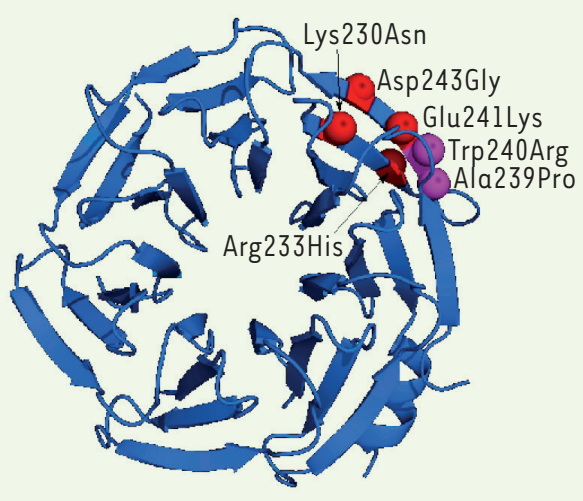

B

IFN $\beta$

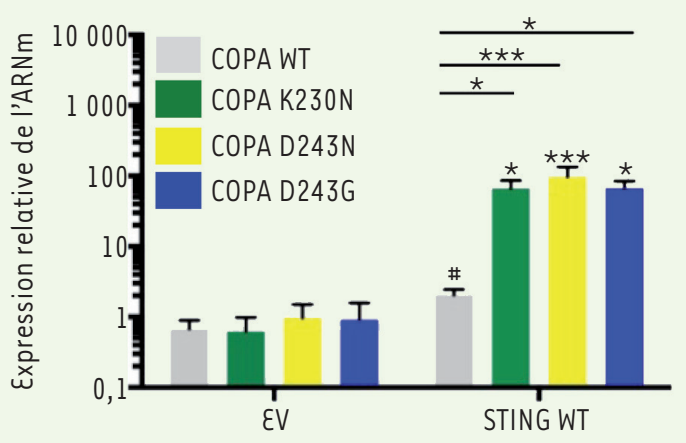

D

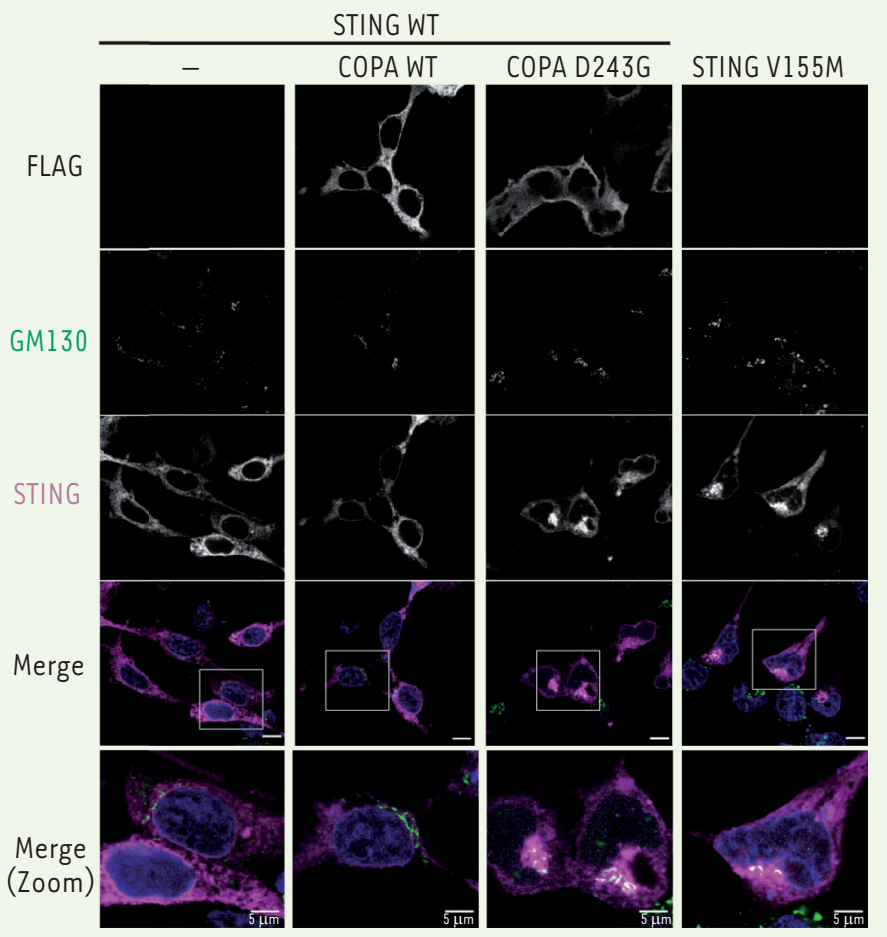

Figure 1. Les mutations de COPA sont responsables d'une induction chronique et pathogénique des interférons due à un défaut du trafic intracellulaire de STING. A. Comparaison des phénotypes cliniques entre SAVI et le syndrome COPA. Les atteintes pulmonaires, rénales, cutanées et articulaires sont respectivement représentées en bleu, vert, rouge et jaune, et la proportion de patients concernés par ces atteintes est indiquée. PID = pneumopathie interstitielle, HIA = hémorragie intra-alvéolaire. L'astérisque représente un unique patient SAVI ayant une atteinte rénale. B. Analyse quantitative de l'ARNm de l'interféron $\beta$ (IFN $\beta$ ) par qPCR (quantitative polymerase chain reaction) dans des cellules HEK293T co-transfectées avec un vecteur témoin (empty vector, EV) ou produisant STING « sauvage » (wild-type, WT) et un vecteur produisant COPA «sauvage» (WT) ou portant les mutations K230N, D243N ou D243G. C. Structure du domaine WD40 de la protéine COPA et localisation des six mutations pathogéniques décrites chez les patients atteints du syndrome COPA. Nous avons étudié l'effet des mutations Lys230Asn (K230N), Arg233His (R233H), Asp243Asn (D243N) et Asp243Gly (D243G) sur l'activité de la protéine. D. Localisations intracellulaires des protéines STING (magenta) et COPA (FLAG, gris) analysées par immunofluorescence et microscopie confocale dans des cellules HEK293FT co-transfectées produisant COPA « sauvage » (WT) ou COPA portant la mutation D243G, et STING « sauvage » (WT) ou STING portant la mutation activatrice V155M décrite dans le SAVI. L'appareil de Golgi et le noyau sont repérés respectivement par le marqueur GM130 (en vert) et par le marqueur DAPI (en bleu). Sur la superposition des marquages GMI30/STING/DAPI, une colocalisation apparente GMI30/STING apparaît en blanc. Barres d'échelle : $10 \mu \mathrm{m}$ ou $5 \mu \mathrm{m}$ («zoom »). 
nous avons diminué l'expression de COPA en utilisant des ARN interférents ( $\operatorname{sh} A R N$ ), et avons alors observé une activation de la voie IFN. La perte de fonction de COPA reproduit donc l'effet des mutations décrites chez les patients, ce qui permet de conclure à leur effet dominant négatif. De plus, l'activation de la voie IFN est abolie dans des lignées cellulaires rendues génétiquement déficientes en STING, ce qui confirme que la présence de la protéine STING est nécessaire à cette voie de signalisation.

\section{COPA et STING, un tango à trois}

Le coatomère COP I (coat protein I), dont COPA est une sous-unité, intervient dans le recrutement de cargos protéiques dans des vésicules voyageant de manière rétrograde de l'appareil de Golgi au réticulum endoplasmique [6]. Ces cargos protéiques sont habituellement rapatriés, après leur maturation dans l'appareil de Golgi, vers le réticulum endoplasmique pour y assurer leur fonction. La protéine COPA est impliquée dans la reconnaissance directe et le tri des cargos, avec lesquels elle interagit. Les mutations de COPA se situent toutes dans le domaine de reconnaissance des cargos (domaine WD40, Figure 1C).

Puisque le trafic de STING entre le réticulum endoplasmique et l'appareil de Golgi est essentiel pour l'induction d'IFN [1], nous avons fait I'hypothèse que COPA pouvait être nécessaire au retour de STING dans le réticulum endoplasmique, et qu'un défaut de l'activité de COPA entraînerait une accumulation de STING dans l'appareil de Golgi et amplifierait ainsi l'induction d'IFN. Nous avons d'abord montré l'existence d'une interaction entre COPA et STING, par co-immunoprécipitation. Cette interaction est altérée lorsque COPA est mutée, suggérant que STING ne peut plus être recrutée dans les vésicules COP I dans le syndrome COPA. De plus, en utilisant différentes formes tronquées de STING, nous avons montré que la liaison de COPA à STING impliquait les domaines transmembranaires de STING. L'importance de ces domaines dans l'interaction entre STING et COPA nous a surpris puisque COPA est une protéine du cytosol et reconnaît des motifs dilysine présentés par les cargos protéiques sur la face cytosolique de la membrane golgienne [6]. De plus, ces motifs sont très proches de l'extrémité C-terminale des cargos protéiques et STING ne présente aucun des motifs décrits jusqu'à présent. L'existence d'au moins une protéine intermédiaire permettant la liaison entre COPA et STING était donc très probable. Un candidat idéal, STIMI (stromal interaction molecule 1), a été suggéré par des travaux dont les résultats ont été publiés récemment [7]. STIMl est une protéine impliquée dans la signalisation intracellulaire du calcium. Elle est principalement localisée dans le réticulum endoplasmique, possède un motif dilysine, et interagit avec STING pour la retenir dans le réticulum endoplasmique et prévenir une suractivation de sa voie de signalisation. Néanmoins, bien que nous ayons montré l'interaction de STING, STIMl et COPA dans des cellules transfectées, l'interaction entre STING et COPA n'est pas affectée lorsque la production de STIMl est diminuée en utilisant un ARN interférent (siARN).

Trois autres études ont établi un lien entre des mutations de COPA et l'induction d'IFN via STING [8-10]. Deux proposent la protéine transmembranaire SURF4 comme intermédiaire de l'interaction entre COPA et STING. SURF4 (surfeit locus protein 4) est un adaptateur connu entre COPA et certains cargos solubles dans la lumière du réticulum endoplasmique et ne possédant pas de motif dilysine [8]. Les auteurs ont montré que SURF4 pouvait former un complexe avec STING, et qu'une diminution de l'expression de SURF4 altérait l'interaction entre STING et COPA, mais cette interaction n’était cependant pas supprimée, suggérant l'implication d'autres partenaires.

\section{Le voyage de STING, sans retour et sans} frein, vers l'appareil de Golgi quand COPA est mutée

$\varepsilon n$ plus de la perturbation de l'interaction entre COPA et STING, notre hypothèse impliquait une accumulation de STING dans l'appareil de Golgi permettant l'activation constante de sa voie de signalisation [1]. Nous avons donc examiné la localisation intracellulaire de STING par immunofluorescence. Nous avons observé une accumulation de STING dans l'appareil de Golgi uniquement lorsque la protéine COPA est mutée (Figure 1D). Cette localisation anormale de la protéine STING ressemble à son agrégation dans l'appareil de Golgi lorsque elle est activée par son ligand cGAMP ou par les mutations activatrices causant le SAVI (e.g. V155M, Figure 1D) [4]. Nous pensons que ce défaut de localisation causé par la perte d'activité de COPA est dû au non-retour de STING vers son état basal dans le réticulum endoplasmique. Il est toutefois possible que la protéine COPA joue un autre rôle, dans l'adressage de STING vers une voie de dégradation (autophagie) connue pour contribuer au contrôle de la production d'IFN (Figure 2).

Notre étude suggère l'existence d'un trafic constant de la protéine STING du réticulum endoplasmique vers l'appareil de Golgi, sans doute dû à une production basale faible de cGAMP par cGAS, et la nécessité d'un retour permanent de STING au réticulum endoplasmique via COP I pour prévenir l'induction pathogénique d'IFN. Bien que nous ne comprenions pas encore si ce processus d'aller-retours consommateur d'énergie comporte un bénéfice particulier pour la cellule ou s'il est juste une conséquence de la labilité des compartiments intracellulaires, il pourrait rendre compte de la variabilité de pénétrance clinique des mutations de COPA : des niveaux différents d'activation constitutive de STING entre individus les rendraient plus ou moins sensibles au dysfonctionnement de la protéine COPA.

\section{Perspectives thérapeutiques}

Depuis quelques années, une thérapie ciblant la signalisation des IFN est utilisée dans les interféronopathies. II s'agit 


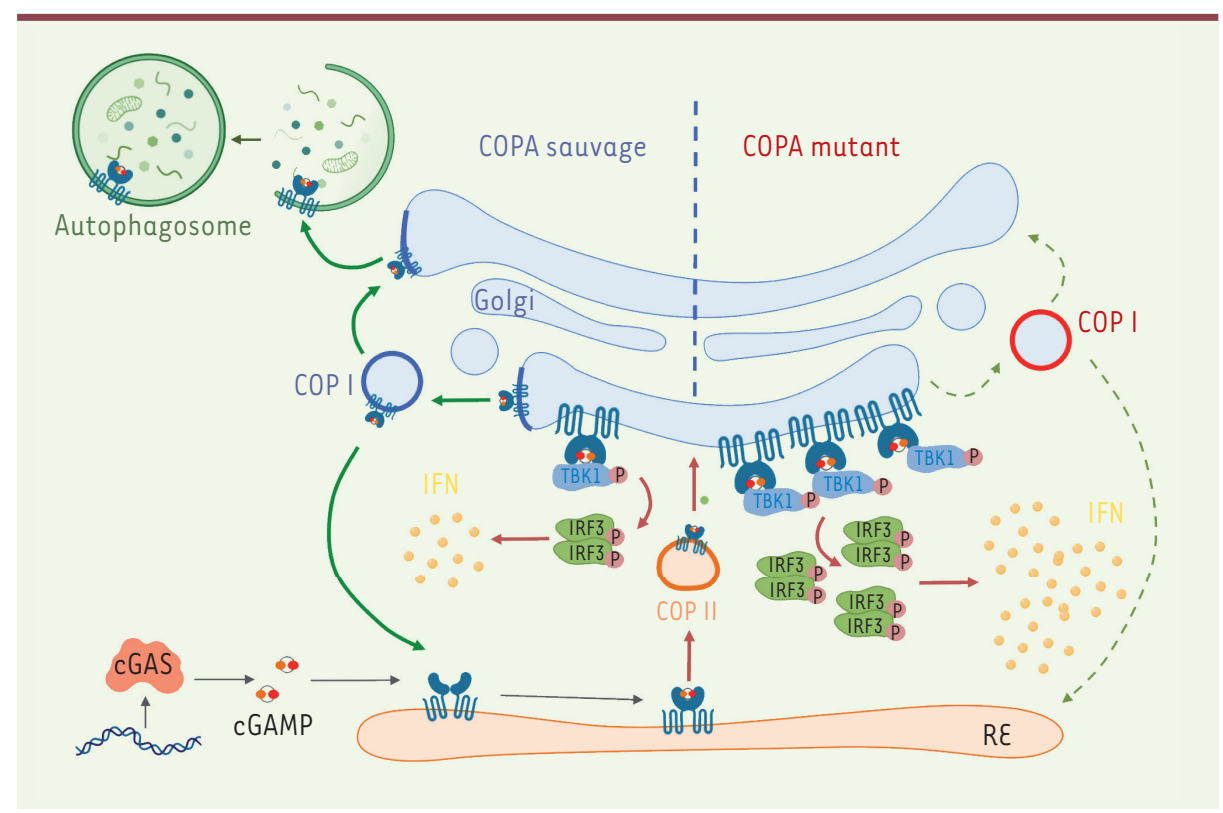

Figure 2. Modèle mécanistique $d u$ rôle de COPA dans le trafic et la signalisation de STING. À gauche: L'ADN libéré par des microorganismes pathogènes ou des cellules abîmées induit la production du second messager cGAMP par l'enzyme cGAS (cyclic AMP-GMP synthase). Après sa liaison au cGAMP, la protéine STING se déplace dans des vésicules antérogrades COP II (coat protein II) vers I'appareil de Golgi, où STING active la phosphorylation de TBKI (TANK-binding kinase 1) et d'IRF3 (interferon regulatory factor 3 ) conduisant à la production des interférons de type 1. La protéine COPA, dans le complexe du coatomère COP I (coat protein I), jouerait un rôle dans le trafic de STING, après signalisation, en lui permettant de retourner dans le réticulum endoplasmique (RE) ou de rejoindre la voie de l'autophagie dans des autophagosomes afin de limiter la signalisation de la voie interféron. Nos résultats indiquent que STING pourrait être un cargo de COP I via COPA. À droite : Lorsque la protéine COPA est mutée dans son domaine WD40, STING ne peut plus être incorporée dans les vésicules COP I, et est maintenue dans l'appareil de Golgi dans un état activé, amplifiant la production d'interféron.

des inhibiteurs de JAKl/2 ou JAKl/3 qui bloquent JAKl, une des deux kinases qui, avec Tyk2, transmettent le signal des IFN jusqu'à leurs gènes cibles. Notre équipe a participé au premier essai clinique de l'utilisation de ces molécules dans le SAVI, dont les résultats sont prometteurs [11]. La démonstration d'une activation constitutive des IFN chez les patients COPA et la compréhension des mécanismes moléculaires impliqués ont déjà encouragé la mise en œuvre, chez trois patients, d'un traitement par un inhibiteur de JAKl/2, qui a entraîné une amélioration notable du phénotype pulmonaire chez l'un [12]. Les résultats de notre étude permettent également d'explorer de nouvelles pistes thérapeutiques. $\diamond$

sCOPing STING out of the Golgi to prevent autoinflammation

\section{REMERCIEMENTS}

Les auteures remercient les patients et leurs familles, leurs collègues, et tous les collaborateurs qui ont permis à ce projet d'aboutir. Les auteures remercient également MSDAVENIR par son projet DevoDecode et la Fondation Square pour leur soutien. Alice Lepelley a bénéficié d'une bourse Marie Curie (Marie SkłodowskaCurie Action Individual Fellowship n 892311).

\section{LIENS D'INTÉRÊT}

Les auteures déclarent n'avoir aucun lien d'intérêt concernant les données publiées dans cet article.

\section{RÉFÉRENCES}

1. Ablasser A, Chen ZJ. cGAS in action: expanding roles in immunity and inflammation. Science $2019 ; 363$.

2. Crow YJ. Type I interferonopathies: a novel set of inborn errors of immunity. Ann NY Acad Sci 2011 ; 1238 : 91-8.

3. Liu Y, Jesus AA, Marrero B, et al. Activated STING in a vascular and pulmonary syndrome. N Engl J Med 2014 ; $371:$ 507-18

4. Jeremiah N, Neven B, Gentili M, et al. Inherited STINGactivating mutation underlies a familial inflammatory syndrome with lupus-like manifestations. J Clin Invest 2014 ; 124 : 5516-20.
5. Volpi S, Tsui J, Mariani M, et al. Type I interferon pathway activation in COPA syndrome. Clin Immunol 2018 ; $187: 33-6$.

6. Brandizzi F, Barlowe C. Organization of the ER-Golgi interface for membrane traffic control. Nat Rev Mol Cell Biol 2013 ; 14 : 382-92.

7. Srikanth $\mathrm{S}$, Woo JS, Wu B, et al. The $\mathrm{Ca}^{2+}$ sensor STIMI regulates the type I interferon response by retaining the signaling adaptor STING at the endoplasmic reticulum. Nat Immunol $2019 ; 20$ : 152-62.

8. Deng Z, Chong Z, Law CS, et al. A defect in COPImediated transport of STING causes immune dysregulation in COPA syndrome. J Exp Med $2020 ; 217$

9. Steiner A, Hrovat Schaale K, Prigione I, et al. Activation of STING due to COPI-deficiency. BioRxiv 2020 ; doi.org/10.1101/2020.07.09.194399.

10. Mukai K, Ogawa $\varepsilon$, Uematsu R, et al. Homeostatic regulation of STING by Golgi-to- $\varepsilon R$ membrane traffic. BioRxiv 2020 ; doi.org/10.1101/2020.05.20.107664.

11. Frémond ML, Rodero MP, Jeremiah N, et al. Efficacy of the Janus kinase $1 / 2$ inhibitor ruxolitinib in the treatment of vasculopathy associated with TMEM173 activating mutations in 3 children. J Allergy Clin Immunol 2016 ; 138 : 1752-5.

12. Frémond ML, Legendre $M$, Fayon $M$, et al. Use of ruxolitinib in COPA syndrome manifesting as lifethreatening alveolar haemorrhage. Thorax $2020 ; 75$ : 92-5.

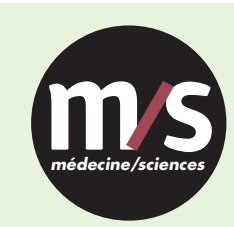

Tarifs d'abonnement $\mathrm{m} / \mathrm{s}-2021$

Abonnez-vous

à médecine/sciences
$>$ Grâce à $m / s$, vivez en direct les progrès des sciences biologiques et médicales

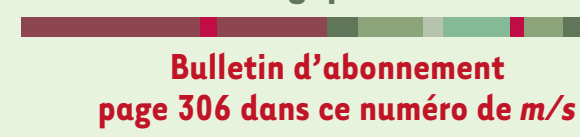

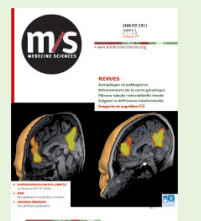

